\title{
FITOPLANCTON Y NUTRIENTES EN EL C.N. DE EL CAMPELLO (ALICANTE)
}

\author{
C. Gomis*,M.J.Sanz ${ }^{*}$ J. Alcober* \& J. Martínez ${ }^{* *}$ \\ * Dpto de Biología Vegetal. Fac. de Ciencias Biológicas. Univ. de Valencia. \\ ** Dpto de Química Analítica. Fac.de Ciencias Químicas. Univ. de Valencia.
}

Palabras clave: Marine phytoplankton, marine coastal, nutrients

\section{ABSTRACT}

\section{PHYTOPLANKTON AND NUTRIENTS IN THE HARBOUR OF EL CAMPELLO}

The phytoplanktonic sequence during an annual cycle in 1985-86, in the Yacht Club of the coast of El Campello (Alicante), was studied. Phytoplankton growth increases during the spring period and probably causes a deplection in nutrients that results in a marked summer decrease in the phytoplanktonic population. Phosphates seem to play a decisive roleas a limitingelement during the whole year; it can beobserved that the populationreaches its maximum when phosphates do, with a short and expected delay. The relatively high values of diversity (3-3,5 bits) in these waters tend to confirm their rather low trophie level.

\section{INTRODUCCIÓN}

Las especies fitoplanctónicas contenidas en una masa de agua están sometidas a cambios continuos en el tiempo, estos cambios progresivos en su composición específica se deben a los efectos diferencialesdelosfactoresfísicos, químicos y biológicos en las especies individuales (Hallegraeff et al., 1986). Con mucha frecuencia los estudios sobre las poblaciones fitoplanctónicas comprueban la posible relación, en cada caso, entre loscambiosde factoresambientales y las poblaciones biológicas que se desarrollan. Este tipo de estudios sobre el fitoplancton en las costas mediterráneas españolas se ha centrado sobre todo en el litoral de las provincias de Barcelona y Castellón.El objetivodel presenteestudioesaportar información al respecto para un puerto de la costa alicantina, el correspondienteala localidad de El Campello.

\section{SITUACIÓN GEOGRÁFICA}

Nuestro punto de muestreo, C.N. de El Campello, está situado en las costas de la provincia de Alicante a $500 \mathrm{~m}$ en dirección norte de la población que le da nombre. Sus coordenadas son $38^{\circ}$ 
25,51 'latitud norte y $00^{\circ} 23,2^{2}$ longitud oeste. Se trata de un puerto deportivo constituido por un malecónde defensa, fundamentalmenteconcebido para los 'Levantes' y por un malecónde cierre que deja una amplia bocana (Figura 1). Ambos maleconesson 'permeables', puestoque no están cementados. Además en la base del malecón de defensa existe un canal, creándose una corriente de renovación bastante eficaz y rápida.

Las muestras se han tomado en los extremos del primer pantalán por hallarse, máso menos, en el centro geométrico del puerto. La profundidad máxima nosupera jamás los $3,5 \mathrm{~m}$, es por ellouna zona que se ve fácilmente afectada por los cambios bruscos de temperatura y el movimiento de embarcaciones.

En estas costas predominan los vientos del noroeste $(21,5 \%)$ seguidos por los del sureste $(19 \%)$ y del este $(18,2 \%)$. A lo largo del año se establece una clara alternancia entre el noroeste y el sureste. De octubre a marzo el predominio corresponde a los vientos del cuarto cuadrante. Por el contrario, de abril a septiembre la supremacía corresponde a los del segundo. Por lo cual durante la primavera y el verano el máximo protagonismo corresponde a vientos marinos, mientras que en otoño es un tiempo de transición en el que los terralesse equilibran con los marinos, alcanzando en invierno los terrales mayor relevancia (Sanjaume, 1985).

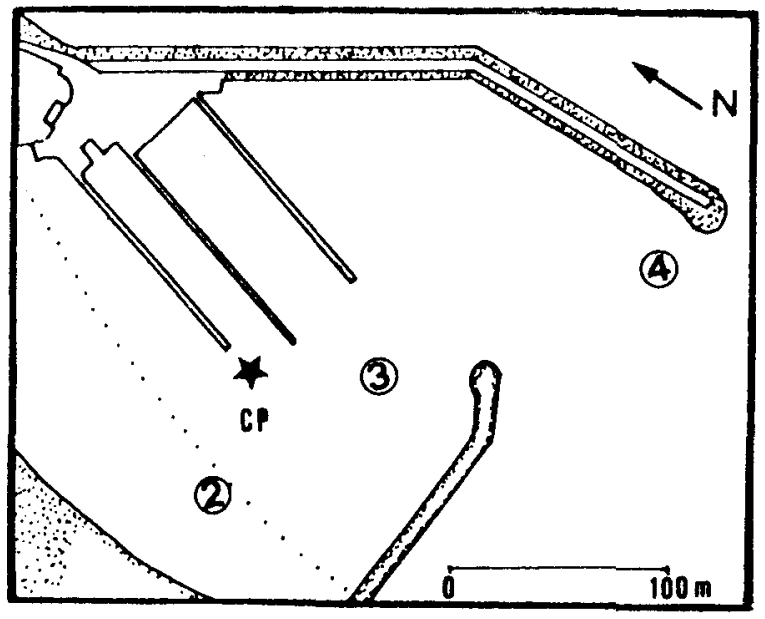

Figura 1.-Situación del punto de muestreo. Situationof sampling station.

\section{MATERIAL Y MÉTODOS}

Se han realizado una serie de muestreosquincenales, de abril de 1986 a febrero de 1987 en el C.N. de El Campello, utilizando una botella hidrográfica Van Dom de 51. Las muestras se recolectaronsiemprea una profundidad que nosuperóen ningún casolos $50 \mathrm{~cm}$ (subsuperficiales).Se medían 'in situ'los siguientes parámetros: temperatura $\left({ }^{\circ} \mathrm{C}\right.$ ), oxígenodisuelto (conun oxímetrode campo WTW Oxi -91 en mg /1), presión (mb), transparencia (utilizando un disco de Secchi estandar), datos sobre el estado de la mar y nubosidad. Inmediatamente a la extracciónde la botella se subdividía el contenido en dos fracciones. Una primera, de aproximadamente 11 se destinaba a los análisis químicos, determinándose las concentraciones en $\mu g r-a t / 1$ de nitritos (según el método de Shinn), nitratos (previa reducción a nitritos)y fosfatossiguiendoel método de Murphy \& Riley (Stricklan \& Parson, 1965). La segunda fracción se fijaba 'in situ' con formol al $4 \%$ saturado de hexametilentetramino según aconseja Sournia (1978), para proceder con posterioridad a la realizaciónde recuentos utilizando un microscopio invertido Olympus IM. Las fotografíasque se muestran en este trabajo se realizaron en el servicio de Microscopía Electronica de la Universidad de Valencia. Los recuentos se realizaron según Uthermohl (Sournia, 1978), sedimentando por término medio unos $200 \mathrm{ml}$ de muestra cada vez. Se contaban al menos 100 individuos de la especie más frecuente (Margalef, 1982). Hemos considerado comoindividuos a cada célula, salvo en el casode Cianoficeasen el que contabamos filamentos o colonias.

Para la identificaciónde los taxonescitados en este trabajo se siguen los criterios de Schiller (1933-37), Husted (1930-66), Cupp (1943), CleveEuler (1951-55), Kofoid (1921), Germain (1981)y Trégouboff (1940). La diversidad se ha calculado utilizando el índice de Shannon-Weaver. 


\section{RESULTADOS Y DISCUSIÓN}

Temperatura, oxígeno, nutrientes y crecimiento fitoplanctónico

Como se puede observar en la Figura 2 existe una clara simetría, con un máximo de temperatura en Agosto $\left(26.8^{\circ} \mathrm{C}\right)$ y un mínimo en Febrero $\left(12.0^{\circ} \mathrm{C}\right)$ que se corresponden con un mínimo $(5.0$ $\mathrm{mgr} / 1)$ y un máximo relativo $(8.6 \mathrm{mgr} / \mathrm{l})$ de oxígeno disuelto (el máximo absoluto se produce en el mes de Enero, con 9.4 mgr/1).

Los valores extremos de las temperaturas vienen a equipararse a los valoresdados por otros autores, en nuestrocasoencontramosun máximo estival en Agosto y un mínimo invernal en Febrero, con una oscilación de $14.8^{\circ} \mathrm{C}$. Bastante próximos a los dados por Herrera \& Margalef (1957) para muestras subsuperficiales en el Puerto de Castellón $\left(25.9^{\circ} \mathrm{Cen}\right.$ Agostoy $12.7^{\circ} \mathrm{C}$ para finales deEnero).Sicomparamosestas temperaturas con las obtenidas por Kim (1979) para Marsella, se observa que los máximos en esta localidad son algo más bajos, 23.6"C.En todo caso se debe considerar que el punto considerado, además de estar a latitudes más bajas, se encuentra en un re-

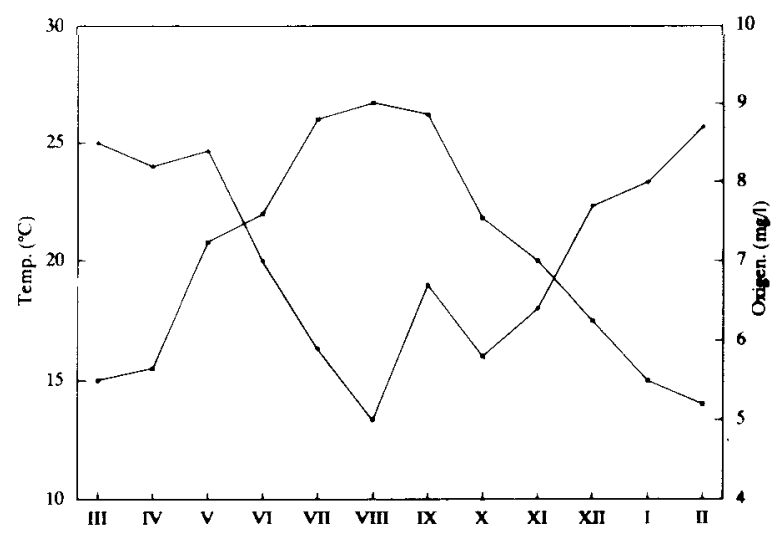

Figura 2.- Valores de temperatura $\left({ }^{\circ} \mathrm{C}\right)$ y oxígeno disuelto (mgr/l).

Temperature values $\left({ }^{\circ} \mathrm{C}\right)$ and disolved oxigen $(\mathrm{mgr} / \mathrm{l})$. cinto en cierta manera protegido y que propicia la obtención de valores más altos.

El máximocrecimientofitoplanctónico(Fig.3) se da en Primavera, para después caer en Verano. La mezcla en la columna de agua en primavera con la consiguiente renovación de nutrientes y el incremento de luz, se traduce en un aumento de la población, este aumento culmina con el agotamiento de los nutrientes en el medio y la población decrece aunque la temperatura siga aumentando. Es entonces cuando se observa un brusco descenso del oxígenodisuelto, debido al aumento de la temperatura y a la disminución de la tasa de fotosíntesis.

Las concentraciones de nitratos oscilan entre $3.36 \mu \mathrm{gr}$-at $/ 1$ y $0.508 \mu \mathrm{gr}$-at $/ 1$, los nitritos aparecen de forma puntual en Diciembre (1.57ygr-at/ 1) y las concentraciones de fosfatos oscilan entre 0.06 y $2.73 \mu \mathrm{gr}$-at/l (Fig. 4).

Se pueden observar dos máximos en la concentración de nitratos, el primero no coincide con elevadas densidades fitoplanctónicas, quizás por que en este caso se puede considerar como limitantela baja concentraciónde fósforo ( $0.5 \mathrm{ygr}$-at/ 1 de fosfatos). Muñoz \& San Fcliu (1972)señalan el fósforo como posible factor limitante de la producción de las Costas de Castellón, Berland et al. (1973) demostraron que los factores limitantes pueden ser clasificados, para las aguas de la Rada de Villefranche-sur-Mer(aguasoligotróficas),de la siguiente forma: fósforo, mezcla de hierro y sustancias acomplejantes, y nitrógeno. En el segundo máximo si se observa una coincidencia, existe un aumento de temperatura, aumentan nitratos y fosfatos traduciéndose en una explosión de la densidad fitoplanctónica que alcanza un máximo que puede considerase absoluto. El desfase de la densidad de la población se debe probablemente a un tiempode respuesta por parte de esta.

El buen tiemposupone un aumento del tráfico de embarcaciones, loque en una zona de tan poca profundidad ocasiona una mezcla de la columna de agua removiendo el sedimento del fondo, fenómeno quese suma a la mezcla provocada por el aumento de temperatura en esta época. Estos hechos se ven reflejados en grandes oscilaciones de la trasparencia en el período veraniego. La 


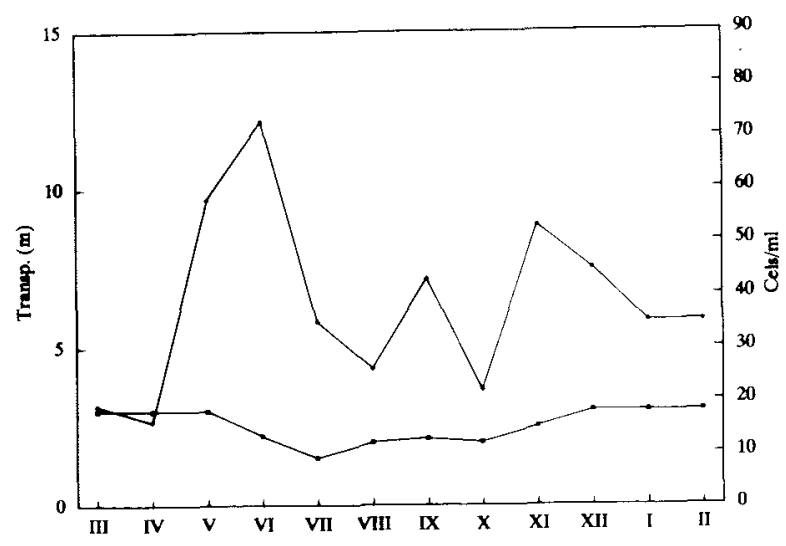

Figura 3.-Valores de trasparencia (m)y densidad fitoplanctónica $(\mathrm{cel} / \mathrm{ml})$.

Values of trasparence $(\mathrm{m})$ and fitoplanktonic density $(\mathrm{cel} / \mathrm{ml})$.

concentraciónde nitritos escasiinapreciabley sólo restringida a los mesesde Verano, loque puede ser debido al aumento de la presión antrópica en las zonas costerasen estos meses que ocasiona un aumento de los vertidos de residuos al mar.

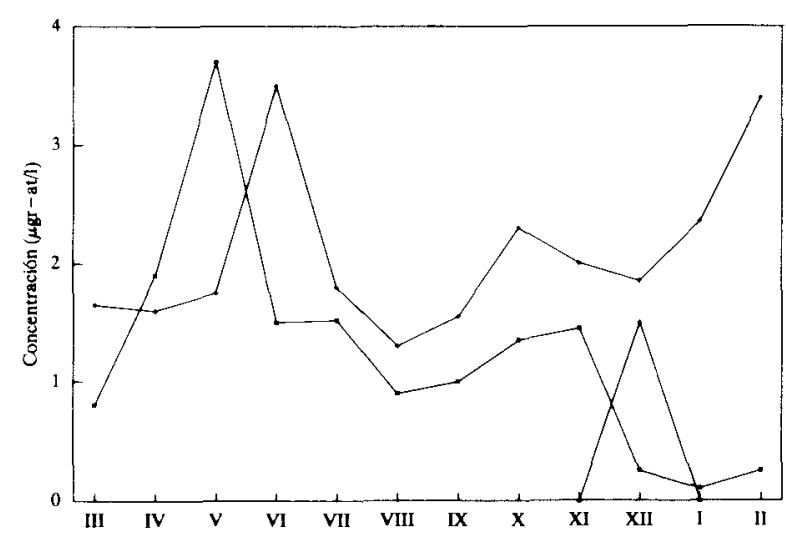

Figura 4.- Valores de los parámetros químicos medidos en $\mu$ gr-at/l (nitratos, nitritos y fosfatos).

Values of measured chemical parameters in $\mu \mathrm{gr}$-at/l(nitrates, nitrites andphosphates).

\section{Diversidad y distribución temporal}

En la Figura 5 se ha representado la distribución temporal de densidad de población de aquellas especies que suponen el $50 \%$ de la población total. Es de destacar la presencia de ciertas especies del género Chaefoceros que pueden llegar a serdominantesocasionalmente(normalmenteen invierno), y constituir durante breves períodos el grueso de la población $\left(14.0 \times 10^{7} \mathrm{cels} / \mathrm{m}^{2}\right)$.es interesante reseñar la existencia de especies que mantienen una presencia númerica, más o menos, constante en el tiempo, tal es el caso de Licmophora abreviafa,Cocconeis disculus o Nifzschialongissima f. genuina; la primera de todas es una diatomea epilítica o epifítica, con una fase claramente planctónica, estas especies raramente superan los $60.0 \times 10^{7}$ cels $/ \mathrm{m}^{2}$, pero suponen un valor númerico muy superior (a lo largo del año) al de las especies de Chaefoceros, pues su presencia noes ocasional,sino constante. Lepfocylindrus danicus, sibien esbastante constanteen el tiempo, presenta unclaro"bloom" primaveral, durante el cual alcanza valores similaresa los Chaetoceros en Invierno. Amphora coffaeiformis y Scrippsiella trochoidea con un comportamiento similar al de $\mathrm{L}$. abreviafa o $C$. disculus, presentan más definidos sus máximos primaverales $\left(75.0\right.$ y $90.0 \times 10^{7}$ cels / $\mathrm{m}^{2}$ ). Se puede observar cierta tendencia a la alternancia de los gruesos de las densidades en las distintas especies, no coincidiendo en prácticamente ningún muestreo los máximos de más de dos especies simultáneamente.

La máxima diversidad (3.53 bits) la encontramos en el inicio de la Primavera (principios de Marzo), cuando las temperaturas son todavía invemales. A medida que avanza la Primavera se produce un descenso de la diversidad que culminacon el mínimode 1.9bits en Junio.Sufre durante los meses de Verano un aumento progresivo, manteniéndose durante los meses de Octubre y Noviembre. Margalef $(1968,1982)$ considera que los valores de diversidad en las costas oscilan entre 1 y 2.5 bits, y entre 3.5 y 4.5 bits en las zonas oceánicas, también sostiene que ésta es más baja en ecosistemas eutróficos, aumentando cuando éstos son oligotróficos. Ello viene a confirmar en cierto modo lo que se sospechaba; se trata en este 


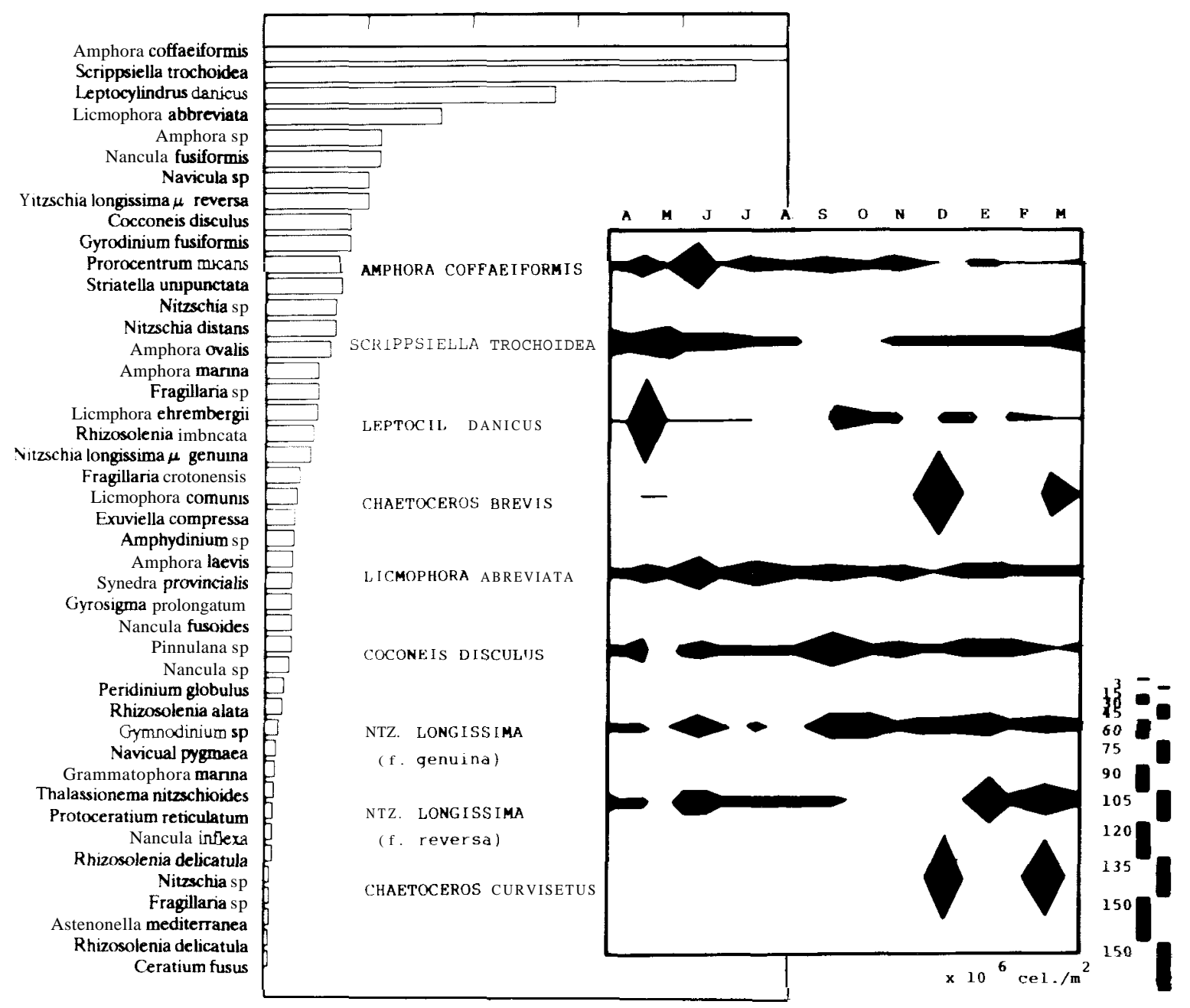

Figura 3.-Media anual de la concentración celular (\% cel/ml) de las especies más abundantes y variación estacionalde las especies más frecuentes en la estación de muestreo.

Annual niean of celular concentration $(\% \mathrm{cel} / \mathrm{ml})$ of species more abundant and seasonal variationof the most frecuent species at the sampling station.

casode aguas relativamente pobres en nutrientes, lo que vine reflejadoen diversidades más altas de las que cabría esperar para aguas neríticas.

Los máximos poblacionales coinciden con los mínimos de diversidad $(74$ cels $/ \mathrm{ml}$ y 1.9 bits de diversidad en la primera quincena de Junio), los crecimientos demográficos se deben normalmente a la proliferación excesiva de pocas espe- cies y no al aumento de especies en el medio. En este caso, son las diatomea Amphora coffaeiformis y el dinoflagelado Scrippsiella frochoidea las dos especiesfundamentalmente responsables del máximo ocurrido en Junio.

En la Figura 6 se muestran algunas especies encontradas en el puerto de El Campello. 


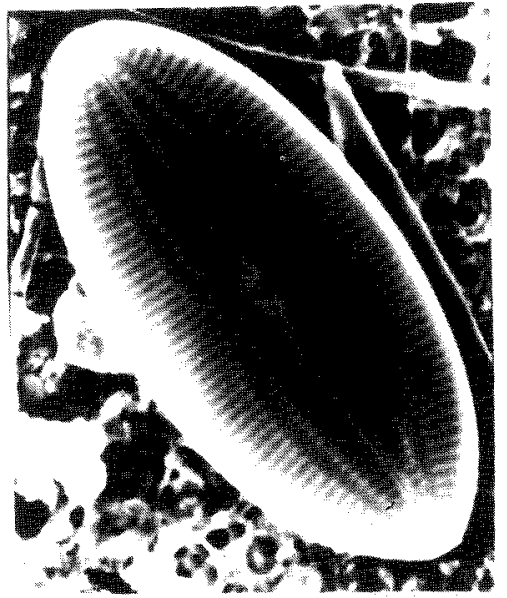

Foto 1

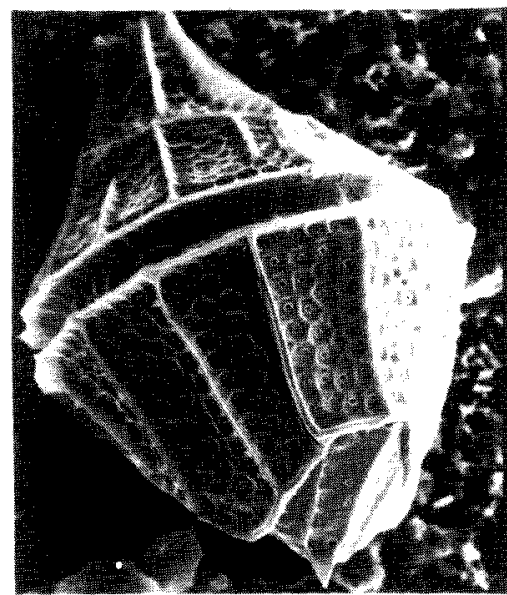

Foto 4

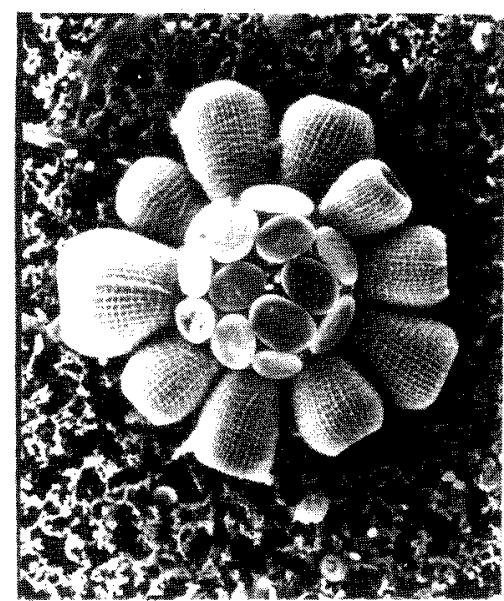

Foto 7

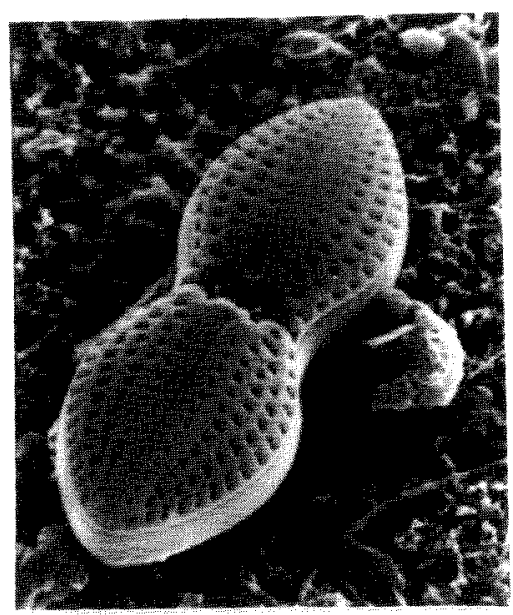

Foto 2

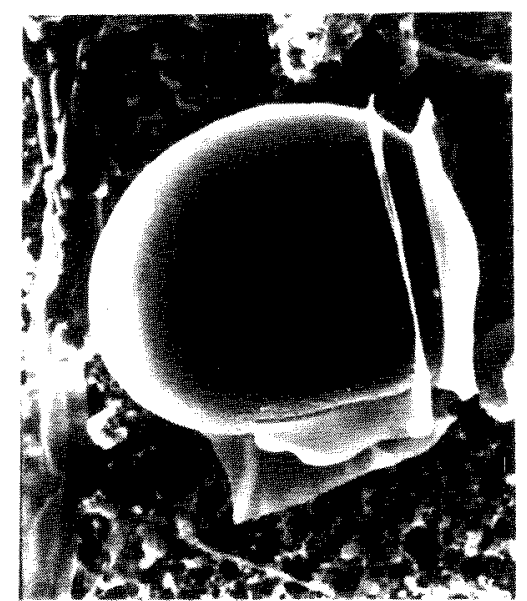

Foto 5

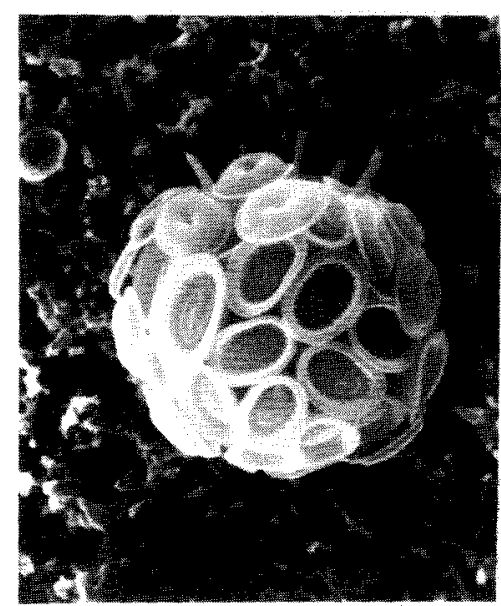

Foto 8

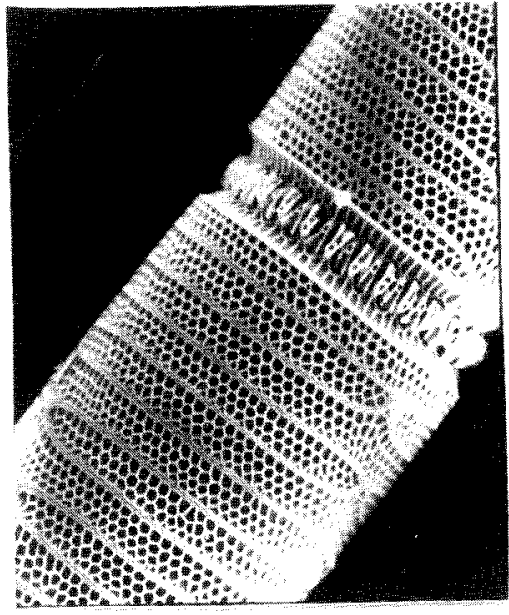

Foto 3

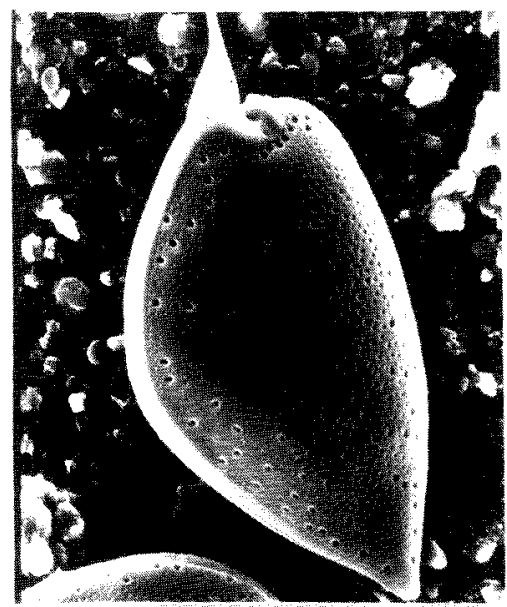

Foto 6

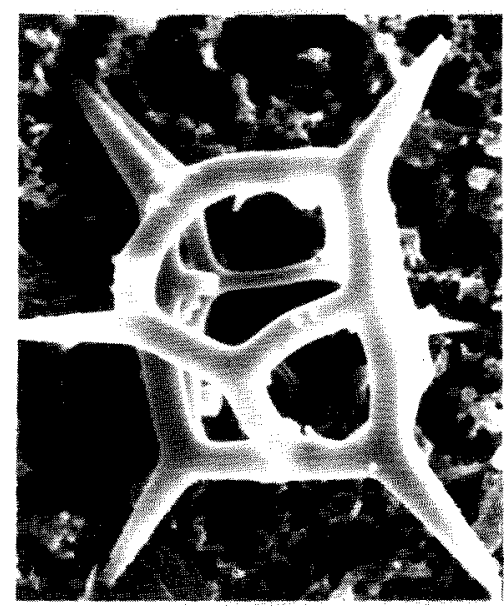

Foto 9 


\section{Bibliografía}

BERLAND, B.R.et al. (1973).Etude de la fertilité des eaux marines au moyen de tests biologiques effectués avec des cultures d'algues. Int. Revue Gesamten Hydrobiol., 58: 473-500.

BourRely,P. 1968,1970,1972. Les algues d'eau douce. 3 vols, Paris. Ed. N. BOUBÉE \& CIE.

Cleve-Euler, A. 1951-55. Die diatomeen von scheweden und Finnland. Kungl Svenska Vetenskademiens Handlingar. BAnd II T.1,2,3,4,5.

Cupp, E.E. 1943. Marine plankton diatoms of the west coast of North America. Bull. Scripps. Ins. Oceanogr., 5: 1-128.

Germain, H. 1981. Flore des Diatomées. Paris. BoubEe $\& \mathrm{CE}$

HallegraefF, G.M.\& Reid, D.D. 1986. Phytoplankton species successions and their hydrological environment at a coastal station of Sydney. Aust. J. Mar. Frcshw. Res., 37:361-377.

Herrera, J. \& Margalef, R. 1957. Hidrografía y fitoplancton de las costas de Castellón, de Juliode 1956 a Juniode 1957. Inv. Pesq., 10:17-44.

Hughes, R.G.1986. Theories and models of species abundancc. The American Naturalist, 128(6): 879899.
KIM, K.-T.1979. Contribution a l'écosystéme pélagique dans les pearges de Carry-le-Rouet. Tethys, 9(2): 149-165.

KoFoID, Ch. \& Swezy, 0.1971. The free living unarmored dinoflagellata. Univ. of California Press.

Margalef, R. 1951. Diversidad de especies en las comunidades naturales. Publ. Inst. Biol. Apl. 9: 527.

- 1985. Western Mediterranean. Oxford Ed. Pergamon Press.

Muñoz, F. \& SAn Feliu, J.M.1972. Hidrografía y fitoplancton de las costas de Castellón de Juliode 1968 a Juniode 1969. Inv. Pesq., 36: 365-392.

SANJAUME, E. 1985. Las costas valencianas. Morfología y sedimentología. Valencia.

Sourvia, A. (ed.) 1978. Phytoplankton Manual. ScorUnesco.

StRicKLAnd, J.D.H. \& Parsons, T.R. 1965. A practica1 handbookof seawateranalisis. Bull.Fish. Res.Can., 167: $1-310$

TrÉgouboff, G. \& Rose, M.1957. Manuel de Planctonologie Méditerranéenne. Tome I. Paris. Centre National de la Recherche Scientifique.

(Pág. anterior)

Figura 6.-Algunas especies observadas al M.E.B.:

Electron micrographs (S.E.M.) of some phy toplanktonic species from El Cainpello harbour.

Foto 1: Navicula pygmaea Kutz. Aparece, generalmente durante los meses estivales. Suele presentar valores de abundancia mínimos. Ârea central en forma de "H".

Foto 2: Diploneis sp. Ehren. Especiede morfología similaa D. interrupta, de acuerdo con las descripcionesde H. Germain (1981), pero de tamaño menor. Ocasionalenel puerto y más o menos constante mar afuera.

Foto 3: Schroederella delicatula (Peragallo) Pavillard. Detalle de la colonia de esta delicada céntrica, no muy frecuente.

Foto 4: Gonyaulax polygrammaStein. Típicoejemplo de Dinoflageladode pared gruesa formada por placas. Para algunos, esta especie, endeterminadas circunstancias, tienecomportamiento tóxico.

Foto 5: Dinophysis paroula (Schüt) Balech. Especie de pequeño tamaño no muy frecuente.

Foto 6: Prorocentrummicans Ehrenberg. De muy amplia distribuciónpornuestras costas. Lo hemosencontrado muy asiduo en aguas eutróficas.

Foto 7: Scyphosphaeraapsteinii Lohmann. Bastante rara, es difícil encontrar ejemplares en buenas condiciones.

Foto 8: Syracosphaera pulchra Lohmann. Bastante corriente, localizada a todos los niveles de profundidad. Perenne.

Foto 9: Dictyocha fibula Ehrenberg. Esqueleto doble de este silicoflagelado, posiblemente tras una divisiónvegetativa. Común durante todo el año. 